\title{
Motivational interviewing \& shared decision making: a primer for audiologists working in a world with more than one right choice
}

\section{Commentary}

Motivational interviewing is a tool used by helping professionals around the world to overcome the natural and sometimes stubborn resistance of individuals with chronic conditions that oftentimes impede living a healthy lifestyle. Conditions such as diabetes, smoking, and alcohol dependency are amenable to motivational interviewing techniques. Several experts have shown MI to be an effective technique for addressing hearing loss in adults. Motivational interviewing (MI) enables audiologists to fortify a person's own motivation and commitment to change or act. For audiologists this means collaborating with patients so that they arrive at making an affirmative decision to accept help and abide by treatment recommended by the audiologist.

Motivational interviewing involves learning and applying some specific conversational techniques. It is possible to spend considerable time and money learning MI techniques. However, it is unrealistic for most audiologists to make this sort of investment in learning this skill. At its core, MI embodies a spirit or attitude that conveys to the patient that "we are in this thing together." Without this spirit of collaboration that acknowledges a person's freedom to make their own decisions, MI conversational techniques are likely to feel manipulative and inauthentic to the patient.

According to the creators of MI, Stephen Rollnick and William Miller, there are four interrelated elements that embody the spirit of MI: collaboration, acceptance, compassion and evocation. When these four elements are combined they position the clinician to be fully engaged in the moment with the patient, actively listening to their concerns and ready to support their plan for success.

Collaboration represents the clinician's ability to recognize that their knowledge and expertise alone is enough to solve the patient's problem. It is an acknowledgment that patients are an expert on their own experiences and our role is supporting them to identify how they will address their problems on their own terms.

Acceptance is the ability of the clinician to acknowledge the person with all their faults and annoyances without judgments of pre-conditions. It is the ability to set aside biases and view patients' behaviors and attitudes from their perspective. To walk a mile in their shoes.

Compassion entails helping the patient do what is in the best interest of the patient, not ourselves. It is our ability to set aside our own personal goals and motivations and do guide patients to what is right for them.

Finally, evocation is the fourth element that embodies the spirit of MI. Evocation is our ability to elicit all the good reasons patients have for their resistance, denial or lack of motivation. Evocation is our ability to not ignore these negative behaviors and maintain a nonjudgmental conversation with the patient about them.
Volume II Issue I - 2019

\author{
Brian Taylor \\ Fuel Medical Group, Resonance Medical, LLC,Academy of \\ Doctors of Audiology, USA
}

Correspondence: Brian Taylor, Fuel Medical Group, Resonance Medical, LLC, Academy of Doctors of Audiology, 4 I I 3 Beverly Ave, USA, Tel 9523933163,Email brian.tylor.aud@gmail.com

Received: December 12, 2018 | Published: January 14, 2019

Detailed courses on MI techniques refer to four stages of a conversation with patients: engage, focus, elicit and plan. Below is a summary of each of these four stages:

Engage: Use of open-ended questions to encourage patients to share their point of view

Focus: Ask follow-up questions that narrows the conversation to the top priorities of the patient

Elicit: Generate solutions from the patient on how to effectively address their priorities

Plan: Setting reasonable treatment goals

\section{Applying MI to clinical audiology}

One approach that embodies the spirit of MI and distills the techniques into a quick, easy-to-apply strategy is to address importance (conviction to act)) and confidence (ability to act effectively) using scaling questions. Recall that scaling questions allow the patient to quantify on a 0 to 10 scale their perception of some trait, such as pain or perception of a problem.

Using this simplified approach, the audiologist would first ask the patient, "On a scale to 0 to 10,0 being not important at all and 10 being very importance, how important is it for you to get help for your hearing?" Generally, if the patient provides you a score of 6 or less, the natural follow-up question would be to ask the patient, "Thanks for your honesty; I am curious why you choose that high of a number, why didn't you say 1 or 0 ?" Prompting the patient in that way makes it very difficult to not have a conversation about problems caused by hearing loss. And, it does it in a way that allows the patient to explore their point of view without interruption from the audiologist.

After encouraging the patient to list some reasons why it might be important to get help for their hearing loss, a second follow-up question can be asked. Asking the patient, "What might it take to move you from a 2 or 3 (any number lower than 6) to a higher number?" enables the patient to think in an aspirational way about their condition. This is the critical moment for patients who are ambivalent or unsure about pursuing treatment. Rather than trying to cajole or convince these patients to get help by talking about the severity of their hearing 
loss, the consequences of their inaction or some other characteristic precluding them to act, giving the patient space to explore their own reasons for taking action to get help unmoors them from the vicious cycle of dithering, denial or indecision - common behaviors associated with hearing loss of gradual onset.

For patients that answer the initial "importance" scaling question with a number higher than 6 , a second scaling question can be asked. The second scaling question addresses the patient's confidence to follow through on treatment adherence (usually hearing aid use) The "confidence" question goes like this, "Let's say you are convinced you are ready to move ahead with hearing aid use and you have no doubt that it's important to do, on scale of 0 to 10,0 being no confidence and 10 complete and total confidence, how confident are you that you can be a successful hearing aid user?" Regardless of the exact number the patient gives you, the natural follow-up question is to ask, "Why did you give that number?" This is another critical moment because it allows the patient to explore their level of confidence and go into some detail about their own situation.

By asking scaling questions about the patient's perception of importance and confidence, along with pertinent follow-up questions the audiologist gains a clearer picture of where the patient stands. For the audiologist the goal of these questions is to first explore importance, followed by confidence. In other words, if the condition or the problem is not important to patients why waste time trying to convince them to act on something they are not ready to do. Further, the efficiency of this approach is that patients tell you what they want to do. Thus, saving time and providing patients with a sense of ownership of their own problem and the solutions required to address it.

Audiologists, because of their training in the medical model of care, often start from a place where they believe they know what is best for patients. However, given the natural reluctance and ambivalence associated with behavior change associated with hearing loss of gradual onset, telling the patient what works best is counterproductive.

Using some MI techniques like the ones outlined here allow audiologists to collaborate and engage with patients on their terms, rather than on the professional's terms.

\section{Shared decision making}

Think about the last time you had to make an emergency purchase of an expensive item. It could have been an air conditioner, a car or maybe even something related to your own health. Depending on the magnitude of the problem, it is likely you were frantically trying to weigh all your options, feverishly surfing the internet, maybe talking with family and friends, gathering as much credible information as you could. These are just some of the factors that make decision making a complex process.

Like motivational interviewing techniques, shared decision making is a valuable tool in an increasingly complex world where individuals must sort through several treatment options. Since we live in a world where most individuals have access to the internet and can conduct a basic Google search for information, audiologists must accept the fact most patients seeking their services know they have an abundance of choices. These choices assume many forms, and include competitive clinics that perform hearing assessments, brick and mortar retail shops and internet-based retail, like Amazon. Since the beginning of the digital era, people with hearing loss have had a range of hearing aid technology options. Regardless of the type of clinic a patient received services, it is likely they had a choice between basic, midlevel and premium hearing aid technology. Today, there are even more choices. As a growing number of high quality non-custom amplification products become available, audiologists must accept that for most patients there is more than one right answer with respect to treatment options.

Because there is often more than one right answer, audiologists must rely on shared decision-making techniques to help patients arrive at a decision on a treatment that fits into their values, budget and lifestyle. Shared-decision making is predicated on the fact that the provider and the patient are working as a team. The objective of this team approach is to enable the patient to decide on a treatment that is best for the individual at this moment in time. It is the relationship between the patient and the provider that drives the shared-decisionmaking process. To better understand the shared-decision process, it helps to contrast it with the transactional approach, which is typically used by audiologists today.

In the transactional approach, the audiologist reviews a few treatment options and makes a recommendation for the option the audiologist thinks would be best for the patient. There is nothing inherently wrong with the transactional approach to decision-making. In fact, there are times when the transactional approach is advised. Some patients may prefer the audiologist take charge and decide for them. Also, if a third-party payer is footing all or most of the bill, it might make sense to move straight to the recommendation in a transactional style.

In most cases, however, patients want to actively participate in the decision-making process. Using a shared-decision making process, it is likely patients will become actively involved in deciding the treatment which is right for them. The first step in this team approach is to have a tacit understanding of the role of each party. The role of the provider is to be the expert on evidence-based information that the patient can integrate into their decision. The role of the patient is to readily share their own priorities. The patient's treatment priorities reflect their values and beliefs.

These roles can be established during the appointment through relationship-centered talk. The process works something like this: At the stage of the appointment where a decision on treatment needs to be made (for audiologists this typically means it has been established the patient is motivated and all pertinent information about their case has been gathered), the audiologist invites the patient (and their communication partner) to be on the same team. The audiologist could say, "Now that we have a clear picture of your hearing loss and have targeted some areas of improvement in communication, we have to decide on a treatment. I know the science about your options, and you are the expert on what matters to you. There are several options, and I'd like to review the advantages and limitations of them. First, I want to find out what matters most to you, so we can work together to find the best solution for you." It's advisable for every audiologist to develop their own talk-track, but the main idea of the previous statement is to communicate to the patient that the decision-making process for a treatment is a team effort and each party brings a clearly defined role to the table.

\section{Treatment options in an audiology clinic}

Rather than simply focus on three or four levels of hearing aid technology, real shared-decision making requires patients be educated on a full range of options. The basic idea is patients must be educated 
before a recommendation can be made. Below are many of the treatment options available from an audiology clinic. For each option, in a shared-decision making paradigm, the audiologist would discuss the advantages and limitations (benefits and risks) for that individual patient. Note that the first option is doing nothing. Given that benign forms of hearing loss are non-life threatening, it's useful to share with patients that doing nothing is an option. However, of course, there are serious risks associated with the options of refusing treatment. Those risks and benefits of refusing treatment must be shared with patients:

a. Decide to do nothing now

b. Non-device options such as auditory training or group aural rehab courses

c. TV or telephone amplifier

d. PSAPs and other hybrid multi-tasking amplifier

e. Hearing aids

f. Hearing system (something broken away from traditional hearing aids to show that some hearing aids have accessories with Bluetooth streaming capability

g. Implantable devices (cochlear implants)

\section{Decision ads and discussing options}

Decision aids are tools that help patients make better understand their treatment options. They can include posters, videos, printed decision trees and online tools. A colorful poster or laminated sheet showing various audiological treatment options can be an effective way to educate patients about the benefits and risks associated with various types of treatment. Decision aids work effectively when the patient can see the options and the audiologist talks through the benefits and limitations (pros and cons) of the various options. During this conversation, encourage the patient to ask questions. Keep in mind, a decision aid, like the one shown in Figure 1 below, is intended to educate the patient. Decision aids don't tell patients what to do, but when integrated into the conversation they help patients gain a firm understanding of the entire range of options and what might be most effective for them.

The goal for the audiologist, when using a decision aid, is to identify what is important for the patient when making a treatment decision. Typically, these factors are cost, cosmetics/appearance, listening demands and ease of use. The decision aid, along with the conversation it generates, usually uncovers what is most important for the patient.

In an audiology practice, most patients will naturally gravitate to the hearing aid solution category. Once the patient has homed in on hearing aids as their treatment preference, the next step in the shared decision-making process is to decide on the level or tier of technology that is most appropriate for the patient. At this point in the process, the focus of the decision-making process is on the style and technology most appropriate for the needs and priorities of the patient.

Recall the role of the audiologist on the decision-making team is to provide the patient with unbiased evidence-based information. For shared decision-making to work effectively, audiologist must have a firm grasp of the peer reviewed research surrounding hearing aid features and benefits and be able to communicate how the results of those studies apply to the hearing aid selection process.

Shared decision-making techniques can be powerful tools in the patient-audiologist relationship, if a few basic principles are followed. First, always seek to educate patients about the benefits and risks associated with each option. Audiologists must use their knowledge of hearing aid features and benefits to provide clear, unbiased information about how each level of technology is expected to benefit the patient. The price of each option should also be shared. Two, limit the number of hearing aid technology options to no more than three. And finally, once a patient understands the pros and cons of their options, ask the patient for their initial preference.

Once the patient has provided you with their initial preference, provide any additional information that could help the patient modify their decision. In a shared decision-making paradigm, moving from an initial preference to a final preference is called "decision talk." For example, after the patient has decided their initial preference is for a basic level of hearing aid technology, the audiologist would inform the patient, using evidence from their own clinical experience and research, of the risks and limitations of their initial choice. Instead of talking about patient options, you are talking more specifically about the pros and cons of their initial decision. After providing this information, the patient can then make a final decision based on what is most important to them.

Shared-decision making is a team effort in which the provider and patient have clearly defined roles. Once patients understand their role, and they have been educated about the benefits and risks of all relevant treatment options, a final decision can be made. Shared decisionmaking using decision aids are an adjunct to effective communication skills. In an era of consumer-driven health care where patients expect to be intimately involved in their own care, there is no replacement for these skills.

\section{Acknowledgements}

The authors appreciate the assistance of all children who participated in this work.

\section{Conflict of interest}

Authors declare that there is no conflict of interest. 